\title{
संगीत में रोज़गार के उपलब्ध क्षेत्र एवं सम्भावनाएं
}

\section{Dr. Shweta Keshri}

Assistant Professor, Kishori Raman Girls P.G. College Mathura

\section{ABSTRACT}

In the presented form, the study of employment related problems faced by students of music has been studied according to the descriptive method of research. An attempt has also been made to throw light on the possible areas of employment in music and what is the role of the current education system in it. Under the current educational system, there are a large number of students in music subjects who have degrees awarded by good universities, but despite having qualifications, there is no employment. In such a situation, the number of students in music is also gradually decreasing. There is only one path left for the students, which is a competition of teaching. While on the contrary there are infinite possibilities in the music, in terms of employment, if a little and necessary changes are included in the teaching system certainly only successful results will be seen. Keywords

Music, Areas of employment in music

\section{सार संक्षेपिका}

प्रस्तुत प्रपत्र में संगीत के विद्यार्थियों के समक्ष आने वाली रोज़गार सम्बन्धी समस्याओं का अध्ययन शोध की विवरणात्मक पद्धति के अनुरूप किया गया है। संगीत विषय में रोज़गार के सम्भावित क्षेत्रों पर और उसमें वर्तमान शिक्षण व्यवस्था की क्या भूमिका है, इस पर भी प्रकाश डालने का प्रयास किया गया है। वर्तमान शिक्षण व्यवस्था के अन्तर्गत संगीत विषय में बहुत अधिक संख्या में ऐसे विद्यार्थी है जिनके पास अच्छे विश्वविद्यालय से सम्मानित डिग्री तो है किन्तु योग्यता होते हुए भी रोज़गार नहीं है। ऐसे में संगीत में विद्यार्थियों की संख्या भी धीरे-धीरे कम होती जा रही हैं। विद्यार्थियों के लिए मात्र एक ही मार्ग शेष रह जाता है वह है शिक्षण का जिसमें की होड़ सी है। जबकि इसके विपरीत संगीत विषय में अनन्त सम्भावनाएं है रोज़गार की दृष्टि से। जिसके अनुरूप यदि शिक्षण प्रणाली में थोड़ा परिवर्तन करते हुए आवश्यक कोर्सेस को सम्मिलित किया जाये तो निश्चित रूप से इसके सफल परिणाम ही देखने को मिलेंगे।

बीज शब्द

संगीत, रोज़गार के क्षेत्र

भूमिका

संगीत के क्षेत्र में जब हम रोज़गार की बात करते है तो निश्चित रूप से हमें शिक्षा व्यवस्था अथवा शिक्षण संस्थानों की बात करना होगा क्योंकि आज एक बड़ा वर्ग इन्हीं शिक्षण संस्थानों में संगीत की शिक्षा प्राप्त कर रहा है। इन शिक्षण संस्थानों व इनमें क्रियान्वित विभिन्न कोर्सेस के माध्यम से जो शिक्षा दी जा रही है सही मायने में वो हमारे समक्ष रोजगार के कितने अवसर प्रदान कर रही है, यह विचारणीय है। इसके साथ ही एक वर्ग ऐसा भी है जो किसी संस्था से न जुड़कर स्वतंत्र रूप से इस दिशा में अग्रसर है, उसके समक्ष भी जीविकोपार्जन के कितने रास्ते हैं और उनके भविष्य निर्माण में शिक्षण संस्थानों की क्या भूमिका है या क्या होनी चाहिए यह भी एक विचारणीय विषय है। अतः प्रस्तुत प्रपत्र में उन सभी बातों की चर्चा की जायेगी जो कि संगीत विषय में रोज़गार का अवसर उपलब्ध करा सकती है और उसमें हमारी शिक्षा व्यवस्था कहां तक सक्षम है। सर्वप्रथम शिक्षण संस्थानों की बात करें तो संगीत विषय में मुख्य रूप से तीन प्रकार के कोर्स देखने को मिलते हैं -

1. डिग्री कोर्स। 


\section{2. सर्टिफिकेट कोर्स।}

3. डिप्लोमा कोर्स।

संगीत विषय में ज्यादातर कोर्स डिग्री कोर्स के रूप में ही देखने को मिलते हैं, जिसके अन्तर्गत स्नातक व परास्नातक की डिग्री प्रदान की जाती है। इनके पाठयक्रम में संगीत के लगभग सभी विषयों को स्नातक व परास्नातक स्तर पर लिया गया है। जबकि सर्टिफिकेट कोर्स, विषय की किसी एक शाखा को लेकर किया जाने वाला स्पेशलाइज्ड कोर्स है व डिप्लोमा कोर्स भी सर्टिफिकेट कोर्स के समान ही किन्तु थोड़ा अधिक गहराई में विषय का अध्ययन करता है। स्नातक तत्पश्चात् परास्नातक के उपरान्त संगीत विषय में एम० फिल० व पी० एच० डी० की उपाधियां भी अनेकों विश्वविद्यालयों में दी जा रही है। (वर्तमान समय में नयी शिक्षा नीति के अन्तर्गत एम० फिल० को सभी विषयों में समाप्त कर दिया गया है।) संगीत विषय में नेट (नेशनल एलिजिबिलिटी टेस्ट) की परीक्षा भी विश्वविद्यालय अनुदान आयोग द्वारा करायी जाती है, जिसे उत्तीर्ण करने के पश्चात् विद्यार्थी प्रवक्ता पद की पात्रता को ग्रहण करता है। कहने का तात्पर्य यह है कि शिक्षा की जिस व्यवस्था में रहकर विद्यार्थी संगीत की शिक्षा ग्रहण करता है वहां जो विकल्प एकमात्र दिखायी पड़ता है वह है शिक्षण का। डिग्री कोर्स के अलावा बहुत कम ही कोर्स डिप्लोमा व सर्टिफिकेट कोर्स के रूप में संगीत विषय के अलग-अलग क्षेत्रों में देखने को मिलती है जैसे कि म्यूज़िक थेरेपी, लोक गीत-संगीत, इन्सट्रूमेन्टेशन तथा किन्हीं वाद्यों के स्पेशलाइज़्ड कोर्स। किन्तु सरकारी संस्थानों में ऐसे बहुत कम ही शिक्षण संस्थान है जहां ये कोर्सेस चलाये जा रहे हैं। जबकि इसके विपरीत कैरियर की दृष्टि से संगीत एक ऐसा विषय है जिसमें की अनेकों सम्भावनाएं है। एक विद्यार्थी संगीत विषय को लेकर अपने भविष्य को कुछ इन रूपों में देख सकता है जैसे परफार्मर, कम्पोज़र, म्युज़िक डायरेक्टर, म्यूज़िक किटिक, साउण्ड रिकॉर्डिस्ट, इवेन्ट प्लानर, लिरिसिस्ट, साउण्ड इंजीनियर, थेरापिस्ट, कमेन्टेटर, रेडियों जॉकी, शो होस्ट व जर्नलिस्ट इत्यादि। किन्तु दुर्भाग्यवश संगीत विषय में जो कोर्स सरकारी शिक्षण संस्थानों में एक विद्यार्थी के समक्ष होते हैं वह उन्हें अधिक मार्ग उपलब्ध नहीं करा पाते हैं। जबकि इसके विपरीत स्नातक व परास्नातक स्तर पर भी यदि इनकी कक्षाएं चलाई जायेंगी तो एक विद्यार्थी को अपनी क्षमता आकने के लिए पर्याप्त समय मिलेगा। अतः संगीत की शिक्षा उस प्रकार से भी दी जानी चाहिए जिससे एक विद्यार्थी स्वयं का आकलन करते हुए स्वयं को उस योग्य बना सके।

कम्पोजिंग, म्यूज़िक डायरेक्शन, साउण्ड इंजीनियरिंग, साउण्ड रिकार्डिस्ट कम्पोजिंग, म्यूज़िक डायरेक्शन, साउण्ड इंजीनियरिंग, साउण्ड रिकार्डिस्ट ऐसे क्षेत्र है जो कि संगीत से सीधा सम्बन्ध रखते हैं अर्थात् यदि इन क्षेत्रों में सर्टिफिकेट व डिप्लोमा कोर्स के अन्तर्गत कोर्स सरकारी शिक्षण संस्थाओं द्वारा चलाये जाये तो निश्चित रूप से एक विद्यार्थी जिनको भी 
इनमें रूचि होगी, अपना भविष्य बना सकते हैं, किन्तु दुर्भाग्यवश ये कोर्सेस सरकारी शिक्षण संस्थानों में न के बराबर है और प्राइवेट इन्स्टीट्यूशन में ये कोर्स बहुत ही महंगे हैं जो कि हर एक के लिए सम्भव नहीं है।

म्यूज़िक क्रिटिक, इवेंट प्लानर, कमेन्टेटर, रेडियो जॉकी, शो होस्ट व जर्नलिज्म

म्यूज़िक क्रिटिक, इवेंट प्लानर, कमेन्टेटर, रेडियो जॉकी, शो होस्ट व जर्नलिज़्म यह कुछ ऐसे क्षेत्र हैं जिसमें कि संगीत से जुड़ा व्यक्ति अधिक अच्छे तरीके से इनमें अपनी भूमिका निभा सकता है। संगीत सीखने वाला व्यक्ति शब्दों के उच्चारण, उसमें काकू भेद अर्थात् आवाज़ के उतार-चढ़ाव आदि को अधिक अच्छी तरह से समझता है, जिससे कि उसके द्वारा कही गयी बात अधिक प्रभावशाली जान पड़ती है। जबकि म्यूज़िक क्रिटिक व जर्नलिस्ट की बात करें तो संगीत जगत में वर्तमान समय में इनकी बहुत अधिक आवश्यकता जान पड़ती है क्योंकि कभी-कभी संगीत का जानकार न होने से भी किसी सांगीतिक कार्यक्रम की रिपोर्टिंग उस स्तर की नहीं हो पाती जैसी कि होना चाहिए। अतः संगीत के यह क्षेत्र बिल्कुल ही रिक्त है, जिनके अनुरूप किन्हीं कोर्सेस के माध्यम से विद्यार्थियों को प्रशिक्षित किया जा सकता है।

शो होस्ट व इवेन्ट प्लानर

कुछ इसी प्रकार से किसी भी सांगीतिक कार्यक्रम के आयोजन में शो होस्ट व इवेन्ट प्लानर की भी बहुत महत्वपूर्ण भूमिका होती है क्योंकि सांगीतिक कार्यक्रमों का संचालन व उस कार्यक्रम के आयोजन में एक कलाकार की दृष्टि से क्या व्यवस्था होनी चाहिए इत्यादि बातों का भी खास ख्याल रखना पड़ता है। अतः यदि इन क्षेत्रों में भी संगीत विषय के अन्तर्गत विषेश कोर्सेस चलाए जाए तो निश्चित रूप से रोजगार के अन्य नये रास्ते खुलेंगे।

म्यूज़िक थेरेपी

म्यूज़िक थेरेपी एक ऐसा क्षेत्र है जिसमें चिकित्सा जगत में संगीत के माध्यम से उपचार कर उसके सफल परिणाम की अनन्त सम्भावनाएँ है। जिस पर कई सफल परीक्षण भी किये जा चुके हैं। ऐसा नहीं है कि ये कोर्स नहीं चलाए जा रहे किन्तु इस कोर्स की जानकारी कम होने व प्रत्येक विश्वविद्यालय में न होने के कारण विद्यार्थी इससे अनभिज्ञ है। भारत वर्ष में मात्र एक-दो संस्थान ही ऐसे है जो कि इस विषय में कोर्स चला रहे है। जबकि इसके विपरीत यह एक ऐसा क्षेत्र है जिसमें कि कार्यक्षेत्र की अनन्त सम्भावनाएं देखी जा सकती है। जैसे - Care Homes, Child Development Centres, Community Spaces, Day Care Centres, School and Nurseries, Prison Services etc.

भारतीय शिक्षा व्यवस्था व शिक्षा नीति की बात करें तो जैसा कि शिक्षा नीति 2019 में भी Integration of Professional Education into higher education and high quality of liberal education की बात कही गयी है अर्थात् शिक्षा में व्यवसायिक शिक्षा का समावेश तथा 
उदार शिक्षा नीति पर बल दिया गया जिसमें विभिन्न विषयों को एक दूसरे से जोड़ते हुए सम्भावनाओं को और अधिक विस्तार देना। इसी सोच को लेकर संगीत को भी अन्य विषयों से जोड़कर रोजगार की नई सम्भावनाओं को जन्म दिया जा सकता है। जैसे-संगीत को विज्ञान से जोड़कर नये क्षेत्र अथवा नये आयाम संगीत में विकसित किये जा सकते हैं। संगीत को चिकित्सा, मनोविज्ञान, भौतिकी से जोड़कर नई सम्भावनाओं को जन्म दिया जा सकता है। संगीत चिकित्सा पद्धति, मनोकित्सक, साउण्ड इंजीनियरिंग, रिकॉर्डिस्ट आदि कुछ ऐसे ही क्षेत्र है।

वहीं दूसरी ओर संगीत विषय में एक और बड़ा वर्ग कलाकार रूप में देखने को मिलता है। संगीत का यह क्षेत्र एक ऐसा क्षेत्र है जिसमें कि संगीत के प्रयोगात्मक पक्ष का गहन अध्ययन व किसी एक परम्परा का निर्वहन करते हुए लम्बे वर्षों का अभ्यास अत्यन्त आवश्यक है। तब जाकर एक लम्बी साधना के पश्चात् कोई भी इस क्षेत्र में अपने भविष्य को रोजगार की दृष्टि से स्थापित कर सकता है। वर्तमान समय की बात करें तो निश्चित रूप से आज का समय शास्त्रीय संगीत के लिए स्वर्णिम युग कहा जा सकता है, जिसमें आज भी शास्त्रीय संगीत के कलाकारों को उनकी कला-प्रतिभा के प्रदर्शन के एवज में सम्मानित पारिश्रमिक दिया जाता है। आज देश की अनेक सामाजिक संस्थाएँ शास्त्रीय संगीत के अनेक कार्यक्रम आयोजित करते हैं, जिसमें देश के अनेक स्थापित व युवा कलाकारों को उनकी प्रतिभा के प्रदर्शन के लिए आमंत्रित किया जाता है। इन सब बातों का यहाँ रखने का अर्थ यह है कि शिक्षण के साथ ही प्रदर्शन भी एक ऐसा क्षेत्र है जिसमें कि संगीत साधकों की संख्या जीवकोपार्जन की दृष्टि से बहुतायत से देखी जा सकती है। बहुत बड़ा वर्ग आज के समय में अपने प्रदर्शन को जीवकोपार्जन का मुख्य स्त्रोत बनाये हुए है। किन्तु प्रश्न यह उठता है कि हमारी शिक्षण प्रणाली की भूमिका एक विद्यार्थी को इस योग्य बनाने में कितनी सक्षम है कि वह प्रदर्शन के क्षेत्र में अपना भविष्य बना सके।

सर्वप्रथम हमें इस बात का पूर्ण आभास होना चाहिए कि विश्वविद्यालय स्तर पर यदि कोई विद्यार्थी अपने विषय के रूप में संगीत विषय का चयन करता है तो निश्चित रूप से उसका लक्ष्य संगीत के क्षेत्र में ही कुछ करने का होता है। अतः उसकी शिक्षा-दीक्षा उस रूप में होनी चाहिए, जिससे कि वह अपने लक्ष्य को प्राप्त करने के लिए पूर्ण रूप से योग्य बन सके।

वर्तमान शिक्षण प्रणाली का आकलन करने पर हम यह पायेंगे कि हमारी शिक्षण प्रणाली एक संगीत के विद्यार्थी को आर्थिक रूप से सबल तो बना सकती है किन्तु एक कलाकार बनने योग्य नहीं। हम जितने भी कलाकारों को सुनते है वे किसी न किसी घराने से संगीत की तालीम लिए होते है। मात्र विद्यालयी शिक्षा के दम पर उनका विकास एक कलाकार के रूप में नहीं हो पाता है। संगीत के क्रियात्मक स्तर को ऊंचा उठाने का श्रेय निशचित रूप से घराना पद्धति को ही जाता है किन्तु घरानों की संकुचित मनोवृत्ति के फलस्वरूप संगीत शिक्षा का विषय कुछ व्यक्ति विशेष के लिए ही रह गया तथा जन साधारण के लिए ये विषय दुर्लभ हो गया क्योंकि घरानें से शिक्षा प्राप्त करने के 
लिए धन की आवश्यकता होती है जिसके आभाव में तो न जाने कितने ही योग्य विद्यार्थी संगीत की शिक्षा से वंचित ही रह जाते हैं। फलस्वरूप कलाकार बन पाना उन विद्यार्थियों के लिए भी दुष्कर हो जाता है जो इसके योग्य होते हैं। अतः एकमात्र अध्यापन का क्षेत्र ही शेष रह जाता है जिसे पाने के लिए विद्यार्थियों में होड़ मची हुई है। आज की शिक्षण प्रणाली ने संगीत का क्षेत्र बहुत ही सीमित कर दिया है, जिसमें नई सम्भावनाओं का मार्ग खोल पाना सम्भव नहीं है। वर्तमान में कार्यरत संगीत के विभिन्न शिक्षण संस्थान हमें डिग्री तो प्रदान कर रही है किन्तु वह योग्यता नहीं, जो एक घराने से सीखे हुए विद्यार्थी में होती है। इसके अतिरिक्त भी कई ऐसे बिन्दु है जो कि इस ओर बाधक है, जैसे सुदृढ़ आधार का आभाव भी विश्वविद्यालयों में विद्यार्थियों के निम्न स्तर का प्रमुख कारण है। यह हमारी शिक्षा व्यवस्था की सबसे बड़ी खामी है जिसका भुगतान विद्यार्थियों को अपने सम्पूर्ण शिक्षण काल में करना पड़ता है।

चूंकि संगीत के क्षेत्र में विद्यार्थियों का एक बड़ा वर्ग इस दिशा में अपना भविष्य बनाना चाहता है अतः यदि शिक्षण संस्थानों के शिक्षण प्रणाली में कुछ अपेक्षीय सुधार किये जाये तो यह विद्यार्थियों के लिए हितकर होगा। जैसा कि हम सभी इस बात से अवगत है कि संगीत एक प्रायोगिक विषय है जिसकी शिक्षा किसी किताब या ग्रंथ से नहीं ग्रहण की जा सकती। यह एक ऐसी विद्या है जो गुरूमुख से ही सीखी जा सकती है किन्तु कक्षा में पर्याप्त समय न होने के कारण सभी विद्यार्थियों पर ध्यान दे पाना व उनसे अलग-अलग सुन कर होने वाली त्रुटियों में सुधार कर पाना प्राय: सम्भव नहीं हो पाता है। अतः संगीत जैसे गुरूमुखी विद्या के लिए गुरू-शिष्य परम्परा या घराना पद्धति ही अधिक उपर्युक्त है। हालांकि शैक्षिक संस्थाओं में संगीत विषय आ जाने से यह जन सुलभ तो हो गया है किन्तु गायन का वह स्तर नहीं रह गया जो कि एक घराने से सीखे हुए विद्यार्थी का होता है। अतः यदि हम विश्वविद्यालयी शिक्षण प्रणाली को घराने का रूप देने का प्रयास करें तो हमें निश्चित रूप से कुछ सफल परिणाम देखने को मिलेंगे। जैसे -

कक्षा को घराने का रूप देते हुए यदि एक ही कक्षा के विद्यार्थियों की शिक्षा एक ही गुरू के सानिध्य में की जाये तो एक विद्यार्थी को गुरू की गायकी का अनुकरण करने एवं एक शिक्षक को अपनी कक्षा के प्रत्येक विद्यार्थी की कमियों व योग्यता को जानने के लिए पर्याप्त अवसर प्राप्त होगा। इसके साथ ही विद्यार्थियों की योग्यता के अनुसार यदि एक ही कक्षा के विद्यार्थियों को अलग-अलग वर्गों में बांट कर उनके स्तर के अनुरूप उन्हें उसी प्रकार की शिक्षा दी जाये तो निश्चित तौर पर कमियों को दूर करने में एक शिक्षक और विद्यार्थी को पर्याप्त समय मिलेगा।

एक घराने की पहचान उसकी कुछ विशेष विशिष्टताओं से होती है जैसे कि स्वर व आवाज़ का लगाव, आलापचारी का ढ़ंग, राग की बढ़त, लय का काम, तानों के प्रकार इत्यादि जो उसे दूसरे घरानों से अलग व विशिष्ट बनाती है। इसी तकनीक का अनुसरण करते हुए रियाज़ करने से कुछ समय पश्चात् विद्यार्थी उसमें निपुण हो जाता है, जो उसकी गायकी में झलकने लगता है। इसका 
अभाव हमें विद्यालयी शिक्षण विधियों में प्रायः देखने को मिलता है अतः इस कमी को दूर करने हेतु शिक्षकों का उद्देश्य मात्र पाठ्यक्रम को पूरा करना न होकर रियाज़ के तरीकों जैसी मूलभूत बातों पर ध्यान देना भी होना चाहिए। शिक्षण के साथ ही बड़ें-बड़ें गायकों एवं वादकों के रिकार्ड भी कक्षा में सुनायें जाने चाहिए, जिससे विद्यार्थियों में रूचि उत्पन्न हो तथा वें उनसे प्रेरणा ले सके। चूंकि गायन के छात्र एवं छात्राओं के स्केल में भी अन्तर होता है अतः दोनों की अलग-अलग कक्षाएं होनी चाहिए जिससे कि गाने में स्केल सम्बन्धी कोई कठिनाई न हो। ऐसी अनेक छोटी-छोटी बातें है जिस पर ध्यान देने की आवश्यकता है।

इसके साथ ही एक महत्वपूर्ण बात यह भी समझनी होगी कि एक अच्छी प्रस्तुती ही एक संगीत के विद्यार्थी की सबसे बड़ी उपलब्धि होती है, जिसके लिए उसको गायन में अच्छा होने के साथ ही कुछ तकनीकी जानकारियों का होना भी आवश्यक है। अतः शिक्षण के साथ-साथ विद्यार्थियों को प्रस्तुती सम्बन्धी बातों की भी शिक्षा दी जानी चाहिए जैसे अपनी आवाज़ के अनुरूप माइक सेटिंग, वॉल्युम सेटिंग तथा गायन को अधिक प्रभावशाली बनाने हेतु इको, डिले, रिवर्ष जैसे तकनीकी अंगों के प्रयोगों का ज्ञान इत्यादि।

इन सब के साथ ही एक अन्य महत्वपूर्ण बात यह है कि संगीत एक प्रायोगिक विषय है जिसमें डी० म्यूज़ का होना नितान्त आवश्यक है, जो कि कुछ वर्षों पूर्व समाप्त कर दिया गया है। क्योंकि साधारणतया यह देखा जाता है कि जो बच्चे प्रयोग में अच्छे होते है उनका शास्त्र पक्ष उतना प्रबल नहीं होता है अतः ऐसे विद्यार्थी डी० म्यूज के माध्यम से शोध कर सकते है। वह आज के समय की जो विकट समरया है कि शोध के कारण सभी का प्रयोग पक्ष कमजोर हो गया है, उस स्थिति से भी बचा जा सकता है।

\section{निष्कर्ष}

शिक्षा की जिस व्यवस्था में रहकर विद्यार्थी संगीत की शिक्षा ग्रहण करता है वहां जो विकल्प एकमात्र दिखायी पड़ता है वह है शिक्षण का। परिणामतः अन्य विषयों की अपेक्षा संगीत के विद्यार्थी प्रायः कम ही देखने को मिलते है। क्योंकि अन्य विषयों की तुलना में संगीत विषय में अपेक्षाकृत रोजगार के कम अवसर उपलब्ध हो पाते है जिसके कारण अन्य अवसरों के तलाश व उसमें अपना भविष्य निर्माण के लिए गैर सरकारी संस्थाओं पर आश्रित होना पड़ता है जो कि अधिक खर्चीला है क्योंकि विश्वविद्यालयों में दी जाने वाली शिक्षा पूर्ण रूप से पर्याप्त नहीं होती कि एक विद्यार्थी को उसमें निपुण बना सके। साथ ही विद्यार्थियों का गिरता स्तर भी एक इसके दुष्परिणाम के रूप में देखा जा सकता है। अतः यदि संगीत जैसी अनन्त सम्भावनाओं के विषय में अन्य सम्भावित क्षेत्रों की ओर भी दृष्टिपात किया जाए तो निश्चित रूप से सफल परिणाम देखने को मिलेंगे। 